\title{
A Cost of Carry-Based Framework for the Bitcoin Futures Price Modeling
}

\author{
Yu-Min Lian, Chi-Hung Cheng, Shih-Hsun Lin, Jui-Hsuan Lin \\ Department of Business Administration, Fu Jen Catholic University, Taiwan \\ Email: 140476@mail.fju.edu.tw, 95352506@nccu.edu.tw
}

How to cite this paper: Lian, Y.-M., Cheng, C.-H., Lin, S.-H. and Lin, J.-H. (2019) A Cost of Carry-Based Framework for the Bitcoin Futures Price Modeling. Journal of Mathematical Finance, 9, 42-53. https://doi.org/10.4236/jmf.2019.91004

Received: January 7, 2019

Accepted: February 24, 2019

Published: February 27, 2019

Copyright $\odot 2019$ by author(s) and Scientific Research Publishing Inc. This work is licensed under the Creative Commons Attribution International License (CC BY 4.0).

http://creativecommons.org/licenses/by/4.0/

\begin{abstract}
In this study, we make use of both the specific method of Monte Carlo simulation and the spot-futures parity with the cost of carry to establish a dynamic price model of Bitcoin futures and to conduct the appraisals and numerical analyses. More specifically, the electricity fees and equipment costs are taken into account and the proposed model is thereby built. Numerical results show that various cost factors have significant effects on the Bitcoin futures price. We employ Monte Carlo simulation to approximate the Bitcoin futures price and we use Python to program the computations.
\end{abstract}

\section{Keywords}

Bitcoin Futures Price, Monte Carlo Simulation, Spot-Futures Parity, Cost of Carry, Geometric Brownian Motion

\section{Introduction}

The prototype of Bitcoin was first introduced in the work of Nakamoto (2008) [1], which mainly explained that Bitcoin was a distributed virtual currency and was formed by users of the entire network. Its peer-to-peer technology and the decentralization characteristic can realize that payment can be made online by means of the e-cash system without a central management institution or bank and this can solve the problem of double-spending. In addition, the release and management transaction of Bitcoin are conducted by network collectively and everybody can participate. Amongst these, block chain is the most important shared public ledger of the entire Bitcoin network. The integrity and time sequence of the block chain have to go through the implementation of encryption. All confirmed transactions are included in the value transfer between the Bitcoin wallet in the block chain and broadcast will be conducted between users. Moreover, normally within the following 10 minutes, the network confirmation is ob- 
tained starting when passing the mining flow. Mining is a distributed consensus system that is used in going through the pending transaction included in the block chain to confirm the pending transaction. The compulsory execution time sequence in the block chain protects the neutrality of network and permits different computers to be consistent based on the status of the system. Reviewing the previous studies on Bitcoin prices, Cheah and Fry (2015) [2] model the Bitcoin price using econometric pattern. Compared with most of assets, the authors prove the occurrence of bubbles of speculation on Bitcoin. Ciaian et al. (2016) [3] analyze the traditional and specific determinants of the price formation on Bitcoin, and show those factors are keys of the price change of Bitcoin.

In recent years, Bitcoin has been receiving a lot of attention of investors. This cryptocurrency as an emerging alternative asset is based on the block chain technology and decentralized network. It has an inelastic money supply with a limit of 21,000,000 bitcoins, which is going to be achieved by today's prediction in 2140. There are more than 2000 kinds of cryptocurrency in transaction markets currently; the total market value reaches 8200 billion USD in January, 2018. Because any increase may be followed by dramatic drops for Bitcoin prices, the market participants represent serious concerns for such a bumpy ride. According to the characteristics of high risk and high return on investment of volatile spot price of Bitcoin, therefore, in order to allow investors to have function of hedging and spot price forecast in Bitcoin investment, the trading service of Bitcoin futures was introduced by the Chicago Board Option Exchange (CBOE) on December 10, 2017. In addition, the Chicago Mercantile Exchange (CME) also activated such service on December 18 in the same year. However, what is comparatively special is both COBE and CME are based on contracts using cash settlement. This means on the due date there will be no actual trading of Bitcoin and instead according to the final settlement price, cash is recorded into one of the accounts and yield is realized through price difference. Bitcoin is one of emerging financial asset. Ever since the subprime mortgage crisis, currency of various countries depreciates and US dollar is no longer a strong currency. In order to seek for reliable investment financial commodity, market investors invest in Bitcoin with a decentralized characteristic one after another to prevent against the autocratic control of the government and the supply and demand is reacted by market fluctuation under the market system. Through the publicity of media, the high risk and high return on investment characteristics of Bitcoin receive attention from market investors broadly. Its futures commodity was introduced in 2017 to provide function of hedging of spot Bitcoin and price forecast for market investors. Delivery of Bitcoin futures in cash becomes a new option for Bitcoin investment, as in Hale et al. (2018) [4].

To accompany the hedge demand against high volatility in Bitcoin market scientifically, we dive into futures price modeling for Bitcoin. A correctly priced Bitcoin futures provides the market with price discovery, leverage, transparency, and risk transfer capacities. This study adopts GBM under the risk-neutral probability measure as the benchmark model of Bitcoin futures price. In addition, mul- 
ti-factors included in the GBM according to the original cost carry model are factors that will affect the price of Bitcoin future. Moreover, consideration is also given to electricity fee and equipment cost as the price difference reference factors of spot and futures of Bitcoin and this dynamic model is thereby built. In the risk-neutral world and under the hypotheses that the change in the price of Bitcoin futures can conform to GBM, this study uses Monte Carlo simulation to simulate all possible price changes to seek for its approximated price and to find out factors that will affect the approximated price. Moreover, we study on the relationship of the amplification factor model and the futures price. The result of this study discovers that various cost factors have significant effect on the Bitcoin futures price. We provide investors with findings of expected price thereby achieving the function of evading the spot price risk of Bitcoin futures.

The remainder of this study is organized as follows. Section 2 introduces the model framework. Section 3 presents the dynamic model and numerical method. Section 4 provides the numerical results and discussions. Section 5 concludes this study.

\section{Dynamic Structure}

Uncovering the Bitcoin price process is a necessary step for choosing the appropriate futures price model. Geometric Brownian motion (GBM) is the classical stochastic process under continuous-time setting, it is been used to describe the dynamics of asset price in previous studies. The characteristic is the assumption that asset price parameters are unrelated to time and its model is set as follows:

$$
d S_{t}=\mu S_{t} d t+\sigma S_{t} d W_{t}
$$

The above formularepresents the dynamic path of the asset price within a short time $(d t)$ in the real world, and in which

$S_{t}$ refers to the asset price at time $t$,

$\mu$ refers to the growth rate or return on investment;

$\sigma$ refers to the fluctuation of return on investment;

$W_{t}$ refers to the Wiener process (i.e., Brownian motion) over time.

GBM is applied in financial mathematics to simulate underlying stock price within the framework of Black and Scholes (1973) [5]. This study is to test whether price change of Bitcoin futures has hedging function and the GBM is brought in to be the price dynamic benchmark model. Under the risk-neutral probability measure, due to the characteristic of conversion between martingale and probability measure, $\mu$ in the traditional GBM is converted to the risk-free interest rate $r$. Then, by taking a further step, through the partial differential equation (PDE) of Black-Scholes framework, one can understand that in the risk-neutral environment, the model factor can be changed into objective risk-free interest rate from subjective growth rate, as in Han (2013) [6]. The GBM benchmark model under the risk-neutral probability measure can be given by:

$$
d S_{t}=r S_{t} d t+\sigma S_{t} d W_{t}
$$


where $S_{t}$ is the Bitcoin spot price at time $t, r$ is the risk-free interest rate, and $\sigma$ is the volatility.

Futures prices will change according to the spot price of the underlying asset. However, basis often appears between futures and spot that will result in different spot price and future price of the underlying asset. Apart from the arbitrage space generated from the price difference of the two assets, the function of price mining is also provided. Therefore, for the relationship between these two asset prices, the put-call futures parity proposed by Tucker (1991) [7] can be used. According to the futures price and between the call option and put option, a certain equilibrium relationship can be maintained and this relationship can be used to decide on the theoretical price of index futures. However, the hypotheses of this theory are that there exist no trading cost and tax, no daily settlement risk, loan interest rate is equivalent, option is European-style, position of futures and option can also be carried till the due date of the futures contract.

The cost of carry model is constructed in the arbitrage portfolio demonstration under the hypothesis of perfect markets that is used to express the model of interrelation of time difference between futures prices and spot prices. This means for the futures price, apart from the spot price, consideration has to be given to costs required within the period from carrying the spot till the date of delivery including warehousing cost, transportation cost, interest cost and insurance cost, etc. However, for different assets, the costs of carry are different. In general, the cost of carry includes the disbursement of loan interest disbursement less the yield of that asset. Regarding the cost of carry of consumption asset, apart from the four aforementioned aspects, the convenience yield has to be deducted. Only for the investment asset in the traditional futures commodities, its cost of carry is formed by the sum of the four aforementioned factors. Pindyck (2001) [8] indicates that the rate of convenience on commodity depended on the level of its spot and futures prices, volatility, and the level of inventory. Heaney (2002) [9] proposes the approximated rate of convenience on commodity based on the volatilities of its spot and futures prices, as well as the maturity of futures contract. As the cost of carry of Bitcoin futures is different from the traditional futures that the warehousing and transportation expense are not required, therefore, when using the Bitcoin mining cost to substitute the cost factor of traditional asset, the cost of carry after improvement is closer to the real Bitcoin futures and spot price difference. In this study, we further establish the price dynamics on Bitcoin futures based on the proposed framework for illustrating the superiority of our model via numerical examples.

\section{Futures Price Modeling and Numerical Approximation}

\subsection{Cost of Carry Model}

The price of Bitcoin futures can be affected by some factors. However, this study is of the opinion that traditional GBM can only capture the stochastic change of prices and under the traditional GBM the factor of the cost of carry theory can 
no longer capture the change of Bitcoin futures effectively and as a result the effectiveness of the appraisal tool is limited by this model. As Bitcoin is not a traditional financial asset, we take a further step according to the cost required for mining Bitcoin to provide new factors in order to improve the cost factor of Bitcoin futures price, to improve the cost of carry theory of traditional futures, to revise its cost of carry factors as electricity fee and mining expense, and the rate of convenience yield is also considered that are further incorporated to the GBM for capturing the basis generated due to carry of cost so that the precision of this model can be enhanced. Consequently, the amplified GBM model is formally given by the following:

$$
d S_{t}=(r+E+F-R) S_{t} d t+\sigma S_{t} d W_{t}
$$

under the risk-neutral probability measure, factors of cost of carry are incorporated into the amplified geometric Brownian model, in which $E$ is the electricity fee, $F$ is the equipment expense, $R$ is the rate of convenience yield. Electricity fee is the main variable cost of Bitcoin mining as extensive electricity computation has to be put in during the course of mining. Therefore, this study utilizes the weighted average of Bitcoin quantity generated from various mines in USA as the simulation factor of electricity fee of various states of US. In addition, equipment expense is based on the present high efficiency miner, the "Ant Miner S9" as the example that the price is approximately US $\$ 2000$ and the rate of convenience yield is based on the quantity of Bitcoin produced by the mines multiplied by the spot price.

\subsection{Monte Carlo Simulation}

The famous Monte Carlo simulation is the numerical simulation method that simulation is conducted on the stochastic process of price change of derivative commodity assets so as to obtain the approximated value of the derivative commodity further. Based on the law of large numbers, if the number of times of simulation is increasing, its simulated value can be closer to the actual value, as in Lian et al. (2015) [10], Lian et al. (2016) [11], and Lian and Chen (2018) [12]. This study makes use of Monte Carlo simulation and presumes that the Bitcoin futures price follows the GBM and the model after improvement is used to conduct extensive simulation trial implementation. Here, the Bitcoin futures price $F_{t}\left(S_{t}, T\right)$ at time $t$ for a contract on $S_{t}$ maturing at time $T$ is given by the proposed cost of carry relationship:

$$
F_{t}\left(S_{t}, T\right)=S_{t} \mathrm{e}^{(r+E+F-R)(T-t)}
$$

in which the 1-year US treasury bill rate of $2 \%$ is used as a proxy for the risk-free rate $r, E$ represents the electricity fee, $F$ indicates the equipment expense, and $R$ is the rate of convenience yield. Electricity fee is based on the average electric fee required to mine one Bitcoin in US, the cost of ant miner S9 required to mine one Bitcoin on the average, the individual spot price cost proportion is $E, F$, and $R . R$ is the rate of convenience yield of Bitcoin spot based on the quantity of Bit- 
coin produced multiplied by the Bitcoin spot price. Assuming there are 252 trading days in one year, and then the discretization interval is $1 / 252$, we conduct 100,000 simulations for calculating each Bitcoin futures price. Upon the implementation of simulation of 100,000 times, the path number of 100,000 on Bitcoin futures pricesis obtained and then these are averaged to compare with the result of the Black-Scholes model and the real path of Bitcoin futures prices.

\section{Numerical Results and Analysis}

Figure 1 plots the comparison of Bitcoin futures price and simulated results (with the reference of Figure 2), while Table 1 documents the preset simulation numerical value of various parameters. The green dotted line in Figure 1 is the traditional GBM under risk-neutral measure and the blue strip curve is the expected price generated after the simulation of 100,000 times by the amplified model and the red solid line is the actual historical price trend of Bitcoin futures. The futures data are from Investing. These daily data correspond to the Bitcoin prices and cover the period between 18, December, 2017 and 18, April, 2018. There are a total of 102 observations for the selected futures contract. In the figure it can obviously show that in the traditional GBM, consideration is only given to risk-free interest rate $r$ and its fluctuation degree and cannot effectively predict the price trend. The amplified GBM can capture more price movement in order to provide more precise price forecast. Through empirical findings of this study, when the Bitcoin futures in the GBM is added with electricity fee and equipment cost as the cost to carry factor and can be closer to the actual price of Bitcoin futures through the simulation result of the Monte Carlo simulation, it can capture more precise dynamic price change compared with single GBM.

The RMSE (root means squared error) used in this study is to measure errors on the actual price change by the amplified model and in the scenario analysis, consideration is given to whether the precision of the model can be improved if the numerical values of factors are adjusted. The RMSE is given by the following:

$$
R M S E=\sqrt{\frac{1}{n} \sum_{i=1}^{n}\left(X_{o b s, i}-X_{\text {model }, i}\right)^{2}}
$$

The RMSE of the traditional GBM and the real price is 9419.897 and the RMSE of the amplified model is 2054.559. This shows that when the cost of carry factor is included in the Bitcoin futures in the traditional GBM, the price forecast ability of the model can be improved and can provide investors with a more precise expected price trend, moreover the results are able to achieve the function of risk management and evade drastic fluctuation of Bitcoin futures price earlier.

Table 1. The preset simulation numerical value of various parameters.

\begin{tabular}{ccccccc}
\hline $\boldsymbol{r}$ & $\boldsymbol{\sigma}$ & $\boldsymbol{S}_{0}$ & $\boldsymbol{T}$ & $\boldsymbol{I n} \boldsymbol{\operatorname { l n }} \boldsymbol{1}$ & $\boldsymbol{\operatorname { l n } R}$ \\
\hline 0.02 & 0.9997 & 19,400 & 252 & -1.43829 & -1.84831 & -0.00933 \\
\hline
\end{tabular}




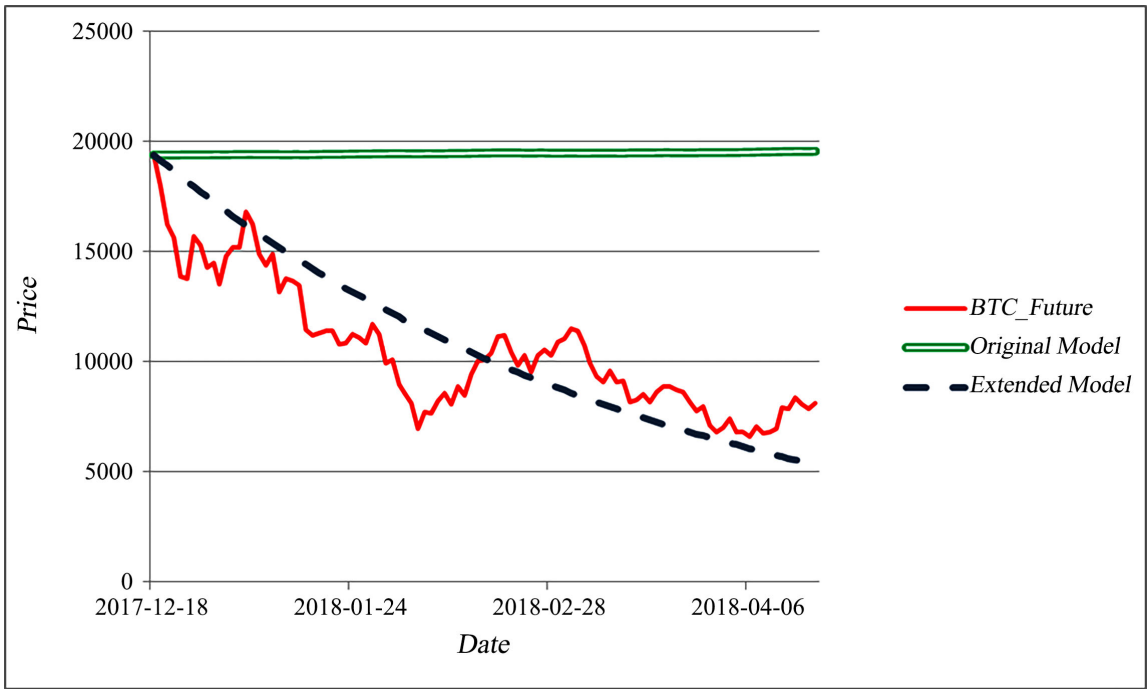

Figure 1. Comparison of Bitcoin futures prices and simulated results.

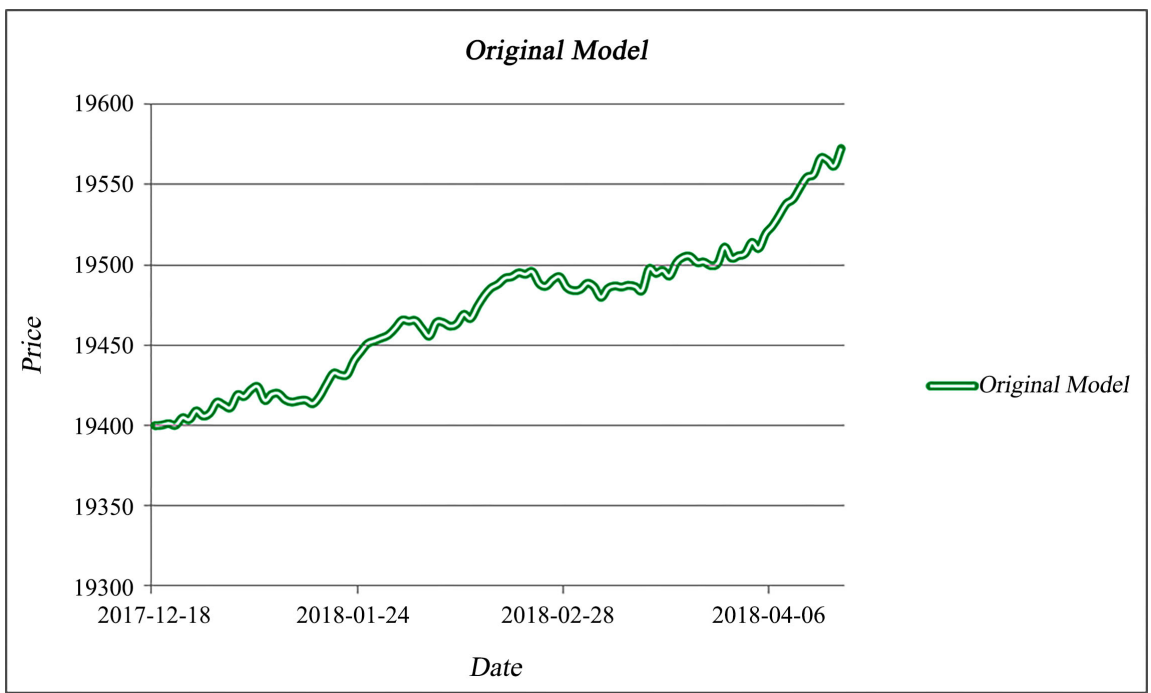

Figure 2. Simulated results of traditional GBM model enlarged the ordinate from Figure 1.

This study further conducts scenario analysis on various factors to check whether change in the numerical value of various factors under the identical risk-neutral probability measure will affect the precision of the model. First the fluctuation rate of $\sigma$ Bitcoin spot historical prices is adjusted as plus and minus $500 \%$ to examine the effect of extreme fluctuation on the model (Figure 3 ). When increasing the fluctuation rate $(+500 \%)$, the change of the simulated expected price is more intense and the finding is the original smooth curve becomes a broken line and one can significantly see the rise and fall change of short term price. Also, the scale of the change trend becomes finer and steeper compared with the original amplified model. However, the scale of expected price change is increased due to the rise of the fluctuation rate and the result is the Bitcoin futures price is overestimated before the beginning of February 2018. In addition, 
the lowering of the fluctuation rate $(-500 \%)$ results in slowdown of risk and the expected price curve of Bitcoin futures also becomes smooth and is quite different from the scale of change in the real price of Bitcoin futures. Summarizing the aforementioned, when the fluctuation rate of Bitcoin spot price is raised, the expected price of Bitcoin futures will change and will be more intense and the price risk is comparatively higher; when lowering the fluctuation rate of the Bitcoin spot price, then the precision rate of the model cannot be enhanced and information obtained is comparatively lower.

When the fluctuation rate is raised (+500\%), the RMSE of its model and real price will rise to 2842.335 and this can show that although the fluctuation trend of short term price can be seen due to over intensive price change, yet it will result in the rise of RMSE value and the forecast precision of the model will be lowered; when the fluctuation rate is lowered (-500\%), the RMSE of its model and real price rises to 2132.267 and this can show that moderating the risk will not produce significant result on the precision of the model.

The natural logarithm of electricity fee plus or minus $20 \%$ where other conditions remain unchanged is acquired to study on whether there is effect on the amplified model due to the rise and fall of the price of electricity fee in different regions (Figure 4). In consideration that the electric fee of various countries is different and under different electricity fee standard, the price of Bitcoin futures will be different. If the electricity is increased by $20 \%$, the entire amplified model will shift downwards. In terms of the view of the buyer's market, the rise of electricity cost will increase the Bitcoin cost and the expected trading price of the market will rise resulting in the fall of quantities required. The incentive to buyer is reduced thereby causing a drop in the Bitcoin price and the entire model will shift downwards; and vice versa, if the electricity fee drops by $20 \%$, the Bitcoin price will rise and the entire model will shift upwards.

Compared with the original amplified model, if the natural logarithm of electricity fee is increased or decreased by $20 \%$, the RMSE of the $20 \%$ increase of the natural logarithm of electricity fee will be 2119.451 ; the RMSE of $20 \%$ decrease of the natural logarithm of electricity fee will be 2092.007. Both of the RMSE values are higher than the original amplified model. Therefore, adjustment on the natural logarithm of electricity fee cannot effectively improve precision.

Under the circumstance of similarity in other conditions, the plus or minus $30 \%$ on the natural logarithm of equipment expense is acquired to explore the environment of price fluctuation of various miner models in the market and the effect on the amplified model (Figure 5). When the equipment cost is increased by $30 \%$, the amplified model will shift downwards. From the view of a miner, an increase in equipment cost will result in increase in the cost of acquiring Bitcoin that will cause reduction in the production volume of Bitcoin mining and the expected price of the market will rise, the price that investors is willing to pay is less and the Bitcoin market price will. This explains that there is significant effect on the model by equipment cost and vice versa, if the equipment cost drops by $30 \%$ the model will shift upwards. 


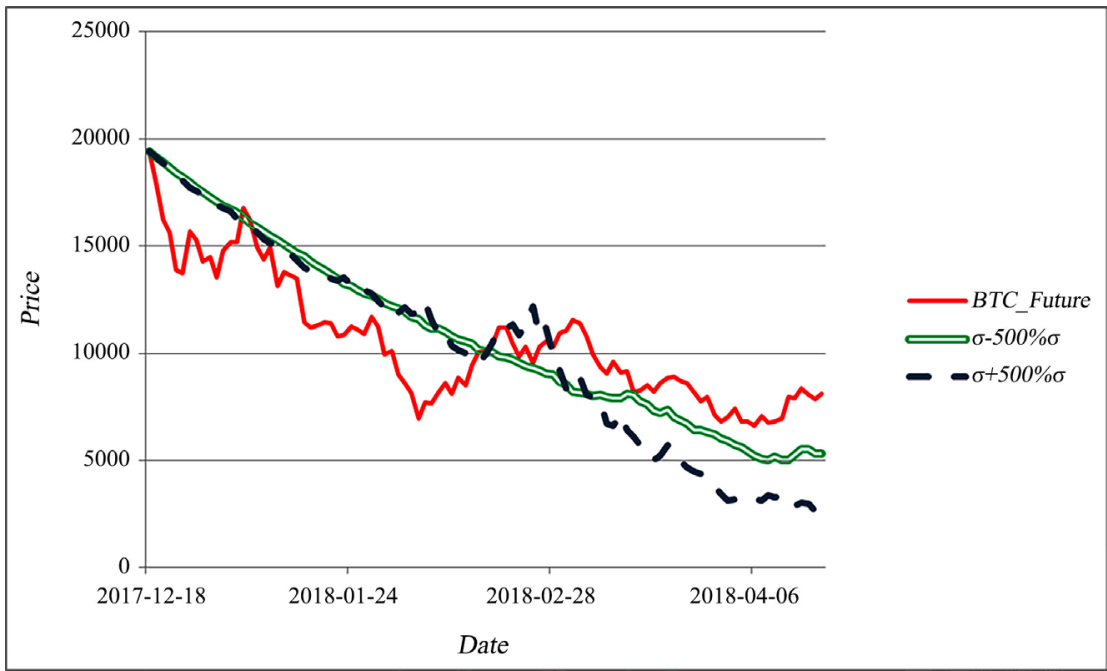

Figure 3. The effect of extreme fluctuation within the model.

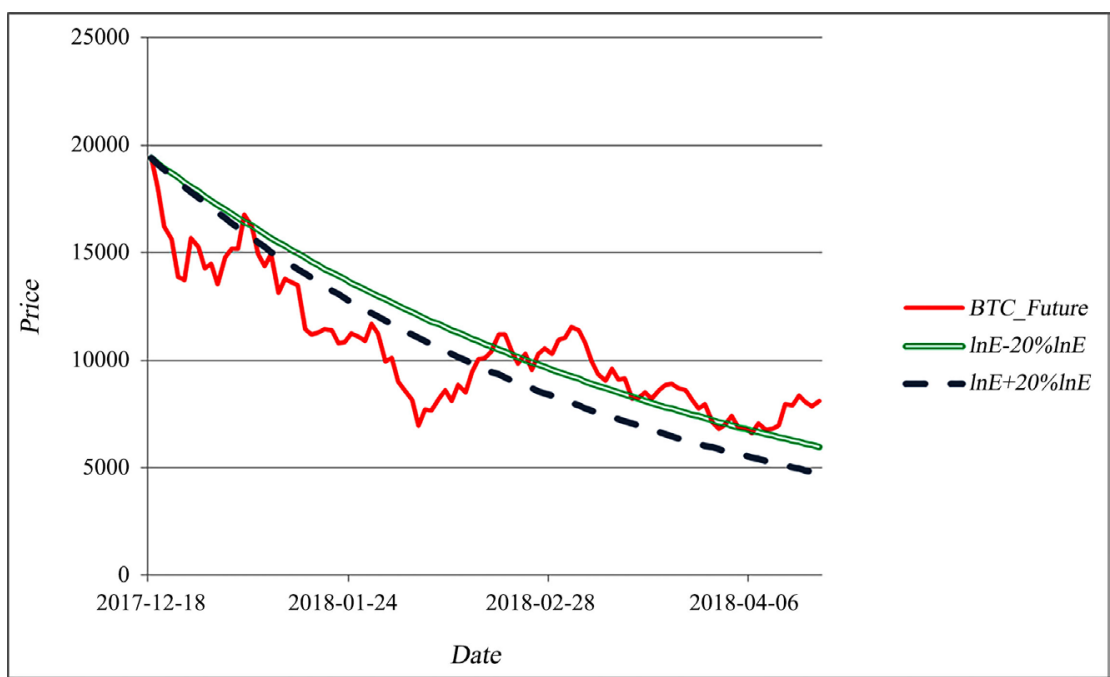

Figure 4. The natural logarithm of electricity fee plus or minus $20 \%$.

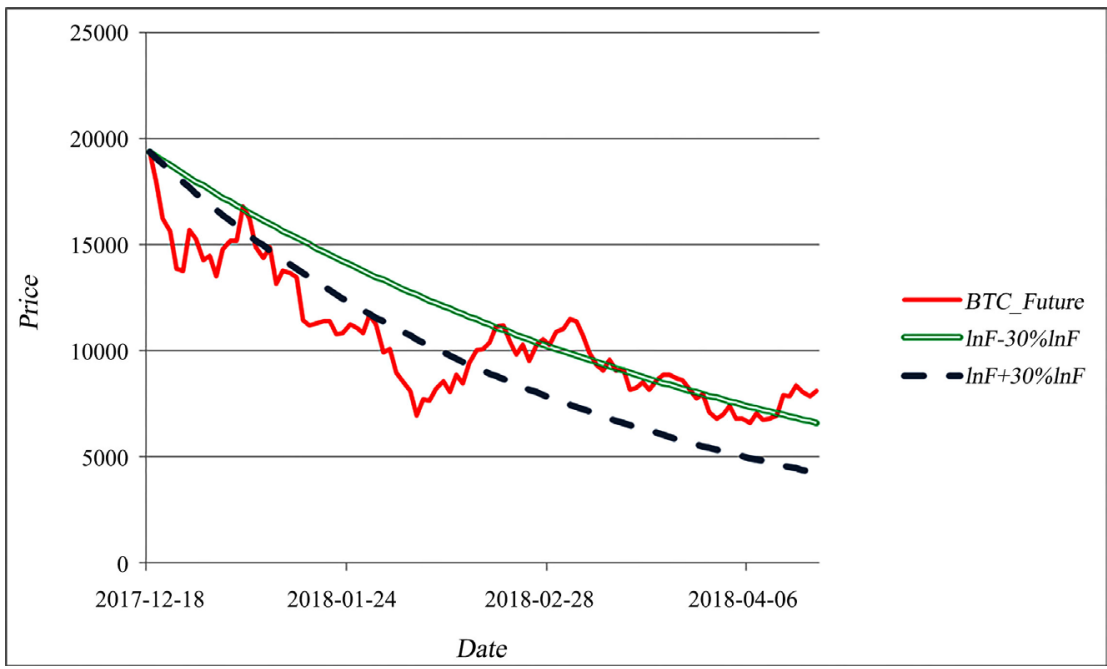

Figure 5. The natural logarithm of equipment expense plus or minus $30 \%$. 
Compared with the original amplified model, if the natural logarithm of equipment expense is increased or decreased by $30 \%$, the RMSE of $30 \%$ increase in the natural logarithm of equipment expense acquired is 2262.691, the RMSE of $30 \%$ decrease in the natural logarithm of equipment expense acquired is 2278.591. The RMSE value above are both higher than the original amplified model, therefore, adjustment on the equipment cost of natural algorithm cannot effectively improve the precision of forecast.

Aiming at adjusting the natural logarithm of the rate of convenience yield by increasing or decreasing $10 \%$ to explore whether the rate of convenience yield of change in trading price in the exchange in different regions will cause effect on the amplified model of Bitcoin futures price (Figure 6), as shown in the figure, the two effects of increase or decrease in the rate of convenience yield is similar and the curves of the simulated expected price of Bitcoin futures coincide. This shows that the result of the effect on the expected price by adjustment on the rate of convenience yield is not significant. If the trading price of the region lower than the rate of convenience yield set by the market average price is $\ln \mathrm{R}-10 \%$, then its RMSE is 2516.047 . The RMSE value is higher than the original amplified GBM model. This shows that if the set parameter lower than the market price is used, the precision of the model will drop and vice versa. If the trading price of the region higher than the rate of convenience yield set by the market average price is $\ln R+10 \%$, then its RMSE is 2148.000 and the RMSE value is higher than the original model.

\section{Conclusions}

In the dynamic model of Bitcoin futures prices, both the electricity fee and the equipment expense are incorporated as the factors of cost of carry and the rate of convenience yield is also considered. Upon simulation on 100,000 times by means

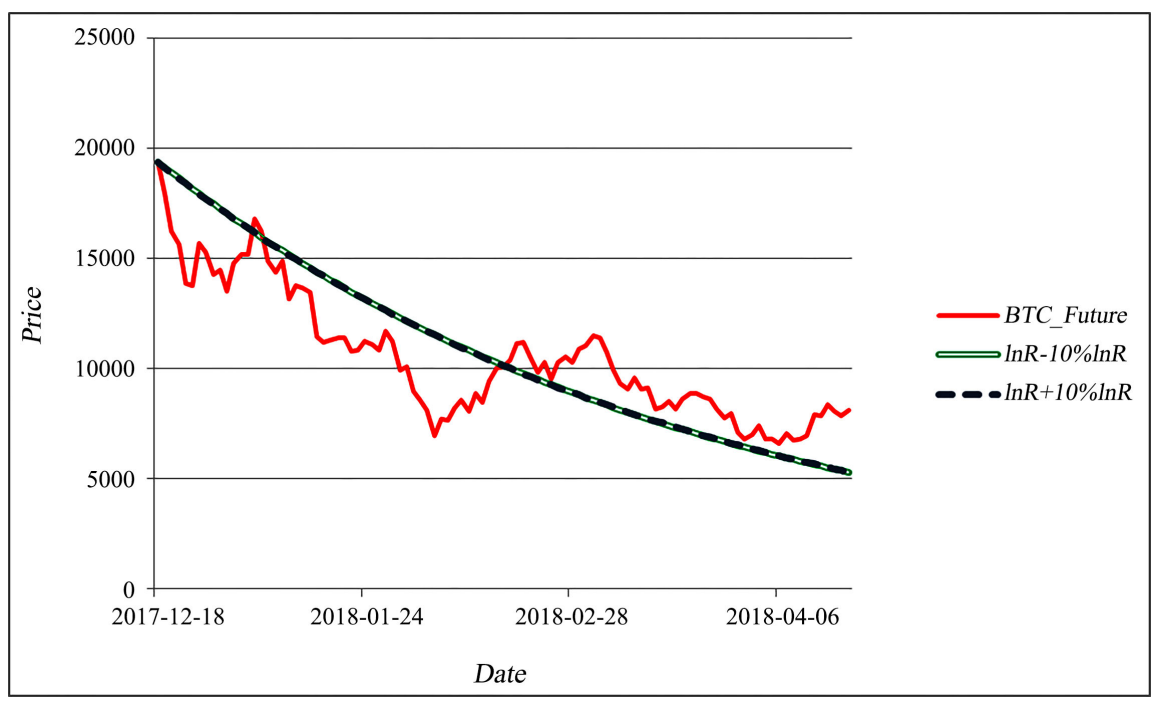

Figure 6. The natural logarithm of the rate of convenience yield by increasing or decreasing $10 \%$. 
of Monte Carlo method, more price movement can be captured so that more precise forecast can be provided to investors. According to this empirical study, it can prove that compared with the traditional GMB, more precise dynamic price change can be captured. This model can provide investors with findings of expected price, transparency of prices can be enhanced, and the function of evading the spot price risk of Bitcoin futures can be achieved.

In scenario analyses, individual factor is adjusted to explore whether change of the factor will affect the precision of the amplified model. When it is adjusted as intensive fluctuation rate, it shows that although the short-term price change can be captured due to increase in the scale of the expected price change, yet, it will also make the error to expand simultaneously. However, reduction of the fluctuation rate will make the reaction of the price of the model not sensitive enough and will become countering to the characteristics of Bitcoin risk and also cannot improve the precision of the improved amplified model. Where the attempt is to change the parameter value of electricity fee, equipment cost and the rate of convenience yield in the amplified model, these three factors cannot reduce the RMSE value through adjustment on the preset parameter value. Therefore, this study is of the opinion that the preset parameter value presumed by the original amplified model can effectively provide an accurate simulation price of the amplified model.

The main contribution of this study is that: we present the market with price discovery, leverage, transparency, and risk transfer capacities based on correctly Bitcoin futures price modeling. In the aspect of function on regulatory, the final target is to reduce the risk of Bitcoin spot market via introducing the corresponding derivative market as an alternative place for market participants.

\section{Acknowledgements}

The authors thank the anonymous referees for helpful comments and suggestions. Yu-Min Lian is grateful for the funding support from the Ministry of Science and Technology under grant MOST107-2410-H-030-001.

\section{Conflicts of Interest}

The authors declare no conflicts of interest regarding the publication of this paper.

\section{References}

[1] Nakamoto, S. (2008) Bitcoin: A Peer-to-Peer Electronic Cash System. https://bitcoin.org/bitcoin.pdf

[2] Cheah, E.-T. and Fry, J. (2015) Speculative Bubbles in Bitcoin Markets? An Empirical Investigation into the Fundamental Value of Bitcoin. Economics Letters, 130, 32-36. https://doi.org/10.1016/j.econlet.2015.02.029

[3] Ciaian, P., Rajcaniova, M. and Kancs, D. (2016) The Economics of Bitcoin Price Formation. Applied Economics, 48, 1799-1815. https://doi.org/10.1080/00036846.2015.1109038 
[4] Hale, G., Krishnamurthy, A., Kudlyak, M. and Shultz, P. (2018) How Futures Trading Changed Bitcoin Prices. FRBSF Economic Letter, Federal Reserve Bank of San Francisco. https://ideas.repec.org/a/fip/fedfel/00163.html

[5] Black, F. and Scholes, M. (1973) The Pricing of Options and Corporate Liabilities, Journal of Political Economy, 81, 637-654. https://doi.org/10.1086/260062

[6] Han, C.H. (2013) Mathematical Problems of Volatility in Finance. Math Media, 37, 26-40.

[7] Tucker, A.L. (1991) Financial Futures, Options, and Swaps. West Publishing Company, St. Paul, MN.

[8] Pindyck, R.S. (2001) The Dynamics of Commodity Spot and Futures Markets: A Primer. The Energy Journal, 22, 1-29. https://doi.org/10.5547/ISSN0195-6574-EJ-Vol22-No3-1

[9] Heaney, R. (2002) Approximation for Convenience Yield in Commodity Futures Pricing. Journal of Futures Markets, 22, 1005-1017. https://doi.org/10.1002/fut.10036

[10] Lian, Y.M., Liao, S.L. and Chen, J.H. (2015) State-Dependent Jump Risks for American Gold Futures Option Pricing. North American Journal of Economics and Finance, 33, 115-133. https://doi.org/10.1016/j.najef.2015.04.001

[11] Lian, Y.M., Chen, J.H. and Liao, S.L. (2016) Option Pricing on Foreign Exchange in a Markov-Modulated, Incomplete-Market Economy. Finance Research Letters, 16, 208-219. https://doi.org/10.1016/j.frl.2015.12.005

[12] Lian, Y.M. and Chen, J.H. (2018) Joint Dynamic Modeling and Option Pricing in Incomplete Derivative-Security Market. Available online by North American Journal of Economics and Finance. 